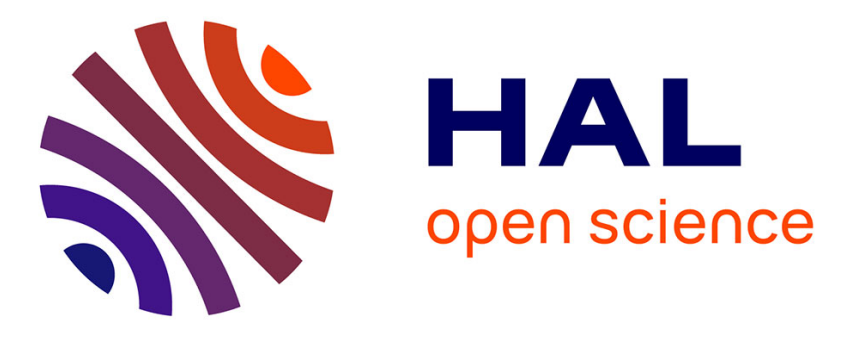

\title{
Bismuth content dependence of the electron spin relaxation time in GaAsBi epilayers and quantum well structures
}

Sawsen Azaizia, Andrea Balocchi, Simone Mazzucato, Fabian Cadiz, S Beato de Le Salle, Henri Lehec, David Lagarde, Alexandre Arnoult, Thierry Amand, Chantal Fontaine, et al.

\section{To cite this version:}

Sawsen Azaizia, Andrea Balocchi, Simone Mazzucato, Fabian Cadiz, S Beato de Le Salle, et al.. Bismuth content dependence of the electron spin relaxation time in GaAsBi epilayers and quantum well structures. Semiconductor Science and Technology, 2018, 33 (11), pp.114013. 10.1088/13616641/aae354 . hal-01944345

\author{
HAL Id: hal-01944345 \\ https://hal.laas.fr/hal-01944345
}

Submitted on 25 Jan 2019

HAL is a multi-disciplinary open access archive for the deposit and dissemination of scientific research documents, whether they are published or not. The documents may come from teaching and research institutions in France or abroad, or from public or private research centers.
L'archive ouverte pluridisciplinaire HAL, est destinée au dépôt et à la diffusion de documents scientifiques de niveau recherche, publiés ou non, émanant des établissements d'enseignement et de recherche français ou étrangers, des laboratoires publics ou privés. 


\section{Bismuth Content Dependence of the Electron Spin Relaxation Time in GaAsBi Epilayers and Quantum Well Structures}

S. Azaizia ${ }^{1}$, A. Balocchi ${ }^{1}$, S. Mazzucato ${ }^{1}$, F. Cadiz $^{1}$, F. Beato de le Salle ${ }^{1}$, H. Lehec ${ }^{1}$, D. Lagarde, A. Arnoult ${ }^{2}, \mathrm{~T}$. Amand ${ }^{1}$, C. Fontaine ${ }^{2}, \mathrm{H}$. Carrère ${ }^{1}$ and X. Marie ${ }^{1}$

${ }^{1}$ Université de Toulouse, INSA-CNRS-UPS, LPCNO, 135 Avenue de Rangueil, 31077 Toulouse, France

${ }^{2}$ LAAS-CNRS, Université de Toulouse, 7 Avenue du Colonel Roche, F-31400 Toulouse, France

Time-resolved optical orientation experiments have been performed in dilute bismide structures. Bulk layers with bismuth fractions in the range 1-3.8\% and quantum wells with bismuth fractions in the range $2.4-7 \%$ were investigated. A clear decrease of the electron spin relaxation time is evidenced in both cases when the bismuth content increases. These results can be well interpreted by the increased efficiency of the spin relaxation mechanisms due to the bismuth induced larger spin-orbit interaction in these alloys.

The incorporation of small concentrations of bismuth (Bi) into GaAs yields a significant reduction of the band gap energy $E_{g}{ }^{1,2}$. As a consequence the dilute bismide alloys, $\mathrm{GaAs}_{1-\mathrm{x}} \mathrm{Bi}_{\mathrm{x}}$, are interesting for potential optical telecommunication or photovoltaic applications $^{3,4}$. As Bismuth is a heavy atom, dilute bismides are also characterized by a much larger spin-orbit interaction compared to $\mathrm{GaAs}^{5}$. Indeed, an increase of the valence band spin-orbit (SO) split-off energy $\Delta_{S O}$ was clearly evidenced in GaAsBi with values reaching $\sim 800 \mathrm{meV}$ and above (i.e. twice the GaAs value) for a bismuth composition of about $10 \%{ }^{6}$. This remarkable property has triggered massive efforts to improve alloy growth, as the condition $\Delta_{S O}>E_{g}$ could lead to a significant reduction of Auger or Inter Valence Band Absorption loss mechanisms in the NIR telecommunication range as suggested by Sweeney's group et $\mathrm{al}^{7,8}$. This system has also been proposed as a good candidate for spintronic applications by Fluegel et al. as the enhanced SO coupling in this material allows for a composition-dependent SO engineering and hence possible electron spin tuneability and manipulation ${ }^{5}$.

For these reasons, the role of the SO interaction on the electron Landé $g$-factor in bulk GaAsBi was studied both theoretically and experimentally ${ }^{9,10}$. It was shown that the introduction of a $\mathrm{Bi}$ fraction of the order of 0.03 led to an increase of the g-factor by a factor 
of 3. Similar observations were made on the exciton $g$-factors in quantum wells in the low temperature range ${ }^{11}$.

Besides, the elctrons spin relaxation time $\tau_{s}$ is a key parameter for spin manipulations as the spin memory should last longer than the manipulation time in order to make use of the electron spin as the information vector. Tong et al. predicted theoretically that the electron spin relaxation time in GaAsBi decreases drastically when the Bi content increases ${ }^{12}$. The experimental investigation of the electron spin relaxation rate in a bulk sample with a bismuth fraction of $2.2 \%$ confirmed these predictions ${ }^{9}$. The result was in good agreement with the characterization of the product $g . \tau_{s}$ (where $g$ is the electron Landé factor) determined by Pursley et al. via Hanle effect measurements ${ }^{13}$. However, a measurement of the bismuth dependence of the electron spin relaxation time is still lacking although it is essential to assess the potential of dilute bismides for spintronic applications. In this work we have measured the electron spin dynamics by time- and polarization- resolved photoluminescence spectroscopy both in GaAsBi bulk and quantum well (QW) structures with various Bi compositions. In both cases we show a clear drop of the electron spin relaxation time when the bismuth content increases, a result in agreement with the increased efficiency of the Dyakonov-Perel mechanism ${ }^{14}$ induced by the stronger spin-orbit interaction.

Dyakonov-Perel mechanism occurs in semiconductors lacking crystal inversion symmetry (to bulk inversion asymmetry - BIA) and is related to the spin splitting of the conduction band induced by the spin-orbit interaction. For wave-vectors $\vec{k} \neq \overrightarrow{0}$, the spin-orbit interaction lifts the spin degeneracy of the conduction band. This BIA induced spin splitting was first pointed out by Dresselhaus ${ }^{15}$. It can be viewed as the result of the action on the electron spin of an effective magnetic field whose amplitude and direction depend on the wave-vector $\vec{k}$ of the electron. The Hamiltonian associated to the interaction writes:

$$
\frac{\hbar}{2} \sigma \Omega(\vec{k})
$$

where $\sigma$ is the Pauli vector. For a given $\vec{k}$, the electron spin will precess around this field with a momentum dependent Larmor vector $\Omega(\vec{k})$.

However, the effective field direction changes after each collision process. As a consequence, spin precession around this field, in intervals between collisions, gives rise to spin relaxation. 
In the usual case of frequent collisions, the relaxation time of the electron spin $\tau_{s}^{i}$ oriented along a direction $i$ can be written:

$\frac{1}{\tau_{s}^{i}}=\left\langle\Omega_{\perp}^{2}\right\rangle \tau_{p}^{*}$

where $\left\langle\Omega_{\perp}^{2}\right\rangle$ is the mean squared component of the precession vector $\Omega$ in the plane orthogonal to the considered spin direction $i$ and $\tau_{p}^{*}$ is the average electron momentum relaxation time. The SO splitting increasing with bismuth content in $\mathrm{GaAsBi}$, we correspondingly expect the electron spin relaxation time to significantly decrease.

In the case of a quantum well, and assuming a Boltzmann distribution of carriers in the conduction band, equation (1) becomes ${ }^{16}$ :

$$
\frac{1}{\tau_{s}^{i}} \sim k_{B} T E_{1}^{2} \gamma_{D}^{2} m^{* 3} \tau_{p}^{*}
$$

Where $E_{1}$ is the first electron confined state energy, $\gamma_{D}$ is the Dresselhaus coefficient and $m^{*}$ is the conduction electron effective mass.

As far as the bulk material is concerned, this latter expression writes ${ }^{17}$ :

$$
\frac{1}{\tau_{s}^{i}} \sim\left(k_{B} T\right)^{3} \gamma_{D}^{2} m^{* 3} \tau_{p}^{*}
$$

Hence the spin relaxation time depends strongly on the confinement and on the electronic band structure of the material through the Dresselhaus coefficient $\gamma_{D}$ and the conduction electron effective mass. It is well established that the electron spin relaxation in un-doped IIIV GaAs-based quantum wells is dominated by the Dyakonov-Perel spin relaxation mechanism ${ }^{16,18,19}$. In bulk III-V materials, depending on the band structure, the doping level and the temperature range, additional mechanisms may contribute to the electron spin relaxation process, namely the Bir-Aronov-Pikus ${ }^{20}$ and Elliott-Yafet ${ }^{21}$ contributions. The BirAronov-Pikus spin relaxation mechanism is due to the electron-hole exchange interaction, and dominates in p-doped samples at low temperatures. Tong et al. have shown theoretically that for undoped bulk GaAs or $\mathrm{GaAsBi}$, the electron spin relaxation time is dominated by the Dyakonov-Perel mechanism ${ }^{12}$, whatever the bismuth fraction is. Additionally, for a bismuth content of $2 \%$, the calculated Dyakonov-Perel electron spin relaxation time is typically two orders of magnitude shorter than the Bir-Aronov-Pikus spin relaxation time, whatever the lattice temperature in the range $50-300 \mathrm{~K}$. The predominance of the Dyakonov-Perel mechanism over the Bir-Aronov-Pikus one is also enhanced when the temperature increases. The Elliott-Yafet mechanism describes the relaxation of electron spins in the conduction band 
due to the spin-orbit coupling via momentum scattering on phonons or impurities. This occurs in small band gap semiconductors with large SO coupling. In GaAsBi alloys the band gap decreases and the SO coupling increases as the bismuth fraction increases. Hence one would expect electron spins in dilute bismides to experience an enhanced Elliott-Yafet relaxation mechanism. However, Litvinenko et al. ${ }^{22}$ and Jiang et al. ${ }^{23,24}$ have shown experimentally and theoretically, respectively, that even in InSb which is characterized by a narrow band gap and a high SO coupling, the Dyakonov-Perel mechanism dominates over the Elliott-Yafet one. Hence, relying on previous studies, and considering the temperature and composition ranges of our study, we expect the spin dynamics in GaAsBi samples to be ruled by the DyakonovPerel relaxation mechanism.

We have studied three GaAsBi/GaAs quantum wells and five GaAsBi bulk samples grown by molecular beam epitaxy. Both types of structures were grown on a (100)-oriented GaAs buffer layer whose growth temperature was set to $580^{\circ} \mathrm{C}$. For the ternary layers (QW and epilayers) a simultaneous opening of $\mathrm{Ga}$ and $\mathrm{Bi}$ cells after cooling down under $\mathrm{As}_{4}$ and a growth temperature in the $360-380^{\circ} \mathrm{C}$ range were used. A nearly stoichiometric V-III ratio and a low $0.3 \mathrm{ml} / \mathrm{s}$ growth rate were also employed ${ }^{25}$. The bismuth compositions and layers thicknesses were provided by high resolution X-ray diffraction measurements, supplemented by secondary ion mass spectrometry analysis for the bulk layers. The bulk samples are pseudomorphically strained $\geq 150 \mathrm{~nm}$ thick layers. The investigated quantum well structures consist of $7.5 \mathrm{~nm}$ quantum wells capped with a 100nm GaAs barrier. The sample characteristics are summarized in table 1.

The samples are optically excited by circularly polarized $\left(\sigma^{+}\right) 1.5$ ps light pulses generated by a mode-locked Ti-Sa laser propagating along the sample growth axis ( $\mathrm{z}$ axis) with a repetition frequency of $80 \mathrm{MHz}$ (average power $P_{\mathrm{exc}}=20 \mathrm{~mW}, 80 \mu \mathrm{m}$ diameter spot). The laser excitation energy is set to $1.57 \mathrm{eV}$.

The dynamics of the photoluminescence (PL) is then recorded using a S1 photocathode streak camera with an overall time resolution of $8 \mathrm{ps}^{9,10}$. The electron spin relaxation time is then extracted from the dynamics of the degree of circular polarization according to $P_{\mathrm{C}}=\left(I^{+}-I^{-}\right.$ )$/\left(I^{+}+I^{-}\right)$, where $I^{+}$and $\Gamma$ represent, respectively, the intensities of the PL co- and counterpolarized with the excitation laser. Since the spin relaxation time of the photo-generated holes is of the order of 1 ps or less for non-resonant excitation in our temperature range ${ }^{26,27,28}$, the 
circular polarization degree of the detected luminescence associated to the fundamental transition corresponds directly to the electron spin polarization degree ${ }^{29}$.

In figure 1, the normalized time-integrated photoluminescence spectra of the quantum well structures and epilayers are presented for $\mathrm{T}=10 \mathrm{~K}$. We observe the redshift of the emission energy when the bismuth content increases, in agreement with previous investigations ${ }^{30,31}$ (in particular see reference 30 and references therein). Spin-orbit interaction was characterized in a previous study carried out on the bulk samples presented here ${ }^{10}$. Photoreflectance measurements showed a strong increase of the SO split-off band, in very good agreement with the energy calculated by tight-binding approach. These results were also in very good agreement with the experimental and theoretical ones reported in the literature. We were not able to probe the SO coupling directly in the quantum well structures, but a number of data for GaAsBi alloys with the same range of composition published in the literature confirm the significant increase of SO coupling ${ }^{8,31}$.

The incorporation of $\mathrm{Bi}$ in the GaAs matrix induces localized states and non-radiative defects. For low Bi content, the PL at low temperature is mainly characterized by the long decay time associated to the localized states (see figure 2(a) for the QW with 2.4\% of bismuth). By increasing the temperature, non-radiative defect channels as well as band-to-band recombination are activated, leading to faster PL decays. As the bismuth content increases, the non-radiative channels gain importance: Even at cryogenic temperatures the PL decay time is now dominated by the recombination on defects with a smaller contribution of the localized states as illustrated in figure 2 (b) for a QW sample with 7\% of bismuth content. In order to minimize excitonic and localization effects in the measurements of the electron spin relaxation time we have performed experiments with lattice temperatures of $100 \mathrm{~K}$ or above and an excitation power of $20 \mathrm{~mW}^{32,33}$.

In figure 3 the time-evolution of the PL circular polarization at $\mathrm{T}=150 \mathrm{~K}$ for the three QW structures is displayed. We observe clearly the decrease of the electron spin relaxation time when the bismuth content increases: it drops from $\tau_{s} \sim 280$ ps for $\mathrm{x}=2.4 \%$ down to 40 ps for $\mathrm{x}=7 \%$. This result is perfectly consistent with the Bi-induced increase of the spin-orbit interaction yielding a more efficient Dyakonov-Perel spin relaxation. A more quantitative comparison with theory is not possible at this stage since this would require a measurement of the electron momentum relaxation rate in each sample. This electron momentum relaxation 
rate may vary slightly as the inhomogeneous broadening changes when the bismuth composition increases. However previous works have evidenced that the electron Hall mobility doesn't vary significantly for samples containing less than $2.5 \%$ bismuth $^{34,35}$, and one study reported an estimated electron mobility of $\sim 2000 \mathrm{~cm}^{2} \mathrm{~V}^{-1} \mathrm{~s}^{-1} 36,37$ for an $8 \%$ bismuth content. These results tend to show that the Hall mobility $\mu_{e}=e \tau_{p} / m^{*}$ is only slightly impacted by $\mathrm{Bi}$ incorporation. It is important to note that the value of the electron effective mass $m^{*}$ is still under debate. Fluegel et al. have shown a slight decrease of $m^{*}$ when the $\mathrm{Bi}$ fraction is increased ${ }^{38}$, whereas Pettinari et al. have observed an increase by $40 \%$ in a $1.7 \% \mathrm{Bi}$ layer $^{39,40}$, compared to the GaAs electron effective mass. However, we first make the assumption that these values do not vary strongly enough to have a major impact on the spin relaxation time in the ternary. On the opposite, the Dresselhaus coefficient $\gamma_{D}$ which appears squared in equation (2) is expected to be 4.4 times larger than in GaAs for a $10 \%$ bismuth content $^{12}$. According to Tong et al., in our concentration range, the calculated bulk Dresselhaus coefficient is expected to increase by a factor of 2 when the bismuth content increases from 2.4 to $7 \%$, suggesting a reduction of the spin relaxation time by a factor of 4 . In our experiment, the spin relaxation time drops from 280 ps to 40 ps, which is a factor of 7 . This discrepancy might find its origin in the fact that the electron states are poorly confined in the quantum well with $2.4 \%$ of bismuth, the band gap reduction impacting mostly the valence band. Indeed, it has been both predicted theoretically and shown experimentally that the conduction band edge of $\mathrm{GaAsBi}$ is lowered by approximately $30 \mathrm{meV} / \% \mathrm{Bi}$ compared to $\mathrm{GaAs}^{41,42,43}$. AlGaAs barriers were used in a $2.2 \%$ GaAsBi QW laser structure in order to overcome the poor electron confinement ${ }^{44}$. Hence, in the $2.4 \%$ quantum well, the electron wavefunction has a larger evanescent component in the GaAs barrier compared to the $7 \%$ quantum well. This yields a slower electron spin relaxation rate in the low-bismuth-content quantum wells due to the GaAs contribution. The temperature dependence of the spin relaxation time is reported in Figure 3(b). For the highest-bismuth-content quantum wells, the electron spin relaxation time is almost independent on the temperature, which is consistent with the Dyakonov-Perel mechanism occurring in quantum wells ${ }^{18}$. For the $2.4 \%$ bismuth sample, the drop in the spin relaxation time when the temperature increases, as proposed above, might be due to the GaAs barrier contribution. 
The PL circular polarization dynamics at $100 \mathrm{~K}$ for the five bulk samples with bismuth fractions varying from $1.16 \%$ to $3.83 \%$ are reported in Figure 4 (a). The drop of the electron spin relaxation time with the bismuth content is again clearly measured. Indeed, the spin relaxation time decreases by about a factor of 10 when the bismuth fraction is increased from 1.16 to $3.83 \%$ at $100 \mathrm{~K}$, and by a factor of 3 at $300 \mathrm{~K}$ as shown in Figure 4 (b). In bulk III-V semiconductor the dominant electron spin relaxation mechanism may depend on the temperature and doping concentrations ${ }^{29}$. According to theoretical calculations ${ }^{12}$, the Dyakonov-Perel mechanism should be dominant at any temperature in bulk GaAsBi and a reduction of the spin relaxation time by a factor of $\sim 2.5$ is expected. Our findings are in reasonable agreement with this prediction for the high temperature range of our experiments, suggesting that Dyakonov-Perel mechanism dominates at $\mathrm{T} 300 \mathrm{~K}$. However, in the intermediate temperature regime $(T \in[100 K ; 250 K])$ we find a much more pronounced drop of the spin relaxation time when the bismuth fraction increases from 1.16 to $3.83 \%$. Moreover the spin relaxation time is almost independent of the temperature for the highest bismuth contents. This feature could suggest that another spin relaxation mechanism such as Elliott-Yafet takes place in the bulk material and becomes dominant for the highest bismuth contents in the low temperature range, due, for instance, to carrier localization ${ }^{45}$ in potential fluctuations arising from Bi clustering ${ }^{46,47,48}$.

If we now compare the spin relaxation time in the quantum well and bulk samples, we observe that the spin relaxation is faster in the bulk. Namely, the spin relaxation time is twice as long in the quantum well with $2.4 \%$ of bismuth than in the $2.34 \%$ bulk for $T \geq 250 \mathrm{~K}$. At a first glance, this is not consistent with a Dyakonov-Perel mechanism occurring in the quantum wells because the confinement effect along the z-axis induces a higher conduction energy and hence a larger spin-orbit splitting in the quantum well than in the bulk material, which should yield a faster spin decay. Again, this behavior can be understood if the electron wavefunction penetration in the GaAs barrier is taken into account, artificially increasing the average spin relaxation time. Finally we mention that the reason of the different initial polarization degree is still not fully understood and further investigations are under way.

Overall, dilute bismides are characterized by fast electron spin decays due to the strong spinorbit coupling in these materials, which at a first glance could be an obstacle for spin manipulation. However, an additional conduction band spin splitting, commonly named 
Rashba term ${ }^{49}$ is present if the confining potential of the heterostructure is asymmetric such as under an external or internal electric field or again if an asymmetric doping is present. This structural inversion asymmetry (SIA) term can interplays with the Dresselhaus one. The high potential of these 2D systems relies on the fact that both BIA and SIA contributions can be tuned independently. When the two contributions cancel each other out, the Dyakonov-Perel spin relaxation mechanism is suppressed, leading to a quench of the electron spin relaxation. This interplay has been demonstrated for instance in (110)-oriented quantum wells: conduction band spin relaxation is strongly suppressed for electron spins oriented along the growth direction and it is greatly increased if a Rahsba contribution is added. ${ }^{50,51,52,53}$. However, in (111)-oriented quantum wells the BIA and SIA terms can cancel each outer out for any spatial directions provided the application of proper electric field. The full electrical control of electron spin relaxation has been demonstrated in GaAs/AlGaAs structures, the BIA term being compensated by the SIA term which arises in the presence of an external applied electric field ${ }^{19,54,55}$. More recently, we have demonstrated that the SIA term can be controlled by the pseudomorphic strain in InGaAs/GaAs quantum wells, without any external electric field, thanks to the piezo-electric property of the (111) orientation ${ }^{56}$. Simmons et al. have shown theoretically that in modulation-doped quantum wells the Rashba term increases significantly in dilute bismide alloys when the bismuth fraction increases due to the respective increase and the decrease of spin-orbit split-off and band gap energies ${ }^{57}$. In GaAsBi, the Rashba term increases faster than the Dresselhaus one as the Bi content increases. This should allow the compensation of the Dresselhaus using weaker electric fields compared to GaAs QWs. Moreover, a partial or complete compensation could be achieved thanks to the built-in piezoelectric field in the case strain and confinement would be correctly balanced, opening the way to spintronic applications.

In conclusion, we have shown that the incorporation of bismuth in GaAs leads to faster electron spin relaxation both in bulk and quantum well structures. In the quantum well structures, the reduced electron spin relaxation time observed when bismuth content increases is consistent with Dyakonov-Perel spin relaxation mechanism whose efficiency is enhanced by the strong spin-orbit coupling in GaAsBi alloy. In bulk structures and in the high temperature range, Dyakonov-Perel also appears to be the dominant spin relaxation 
mechanism. However, for bismuth fractions higher than $2 \%$ in the low temperature range, the experimental data tend to evidence that a competing mechanism arises, potentially being Elliott-Yafet. In light of these results, GaAsBi heterostructures could be good candidates for spintronic applications, provided that crystal orientation and heterostructure symmetry are taken into account in the device designs.

\section{Acknowledgments :}

Part of this work has been performed in the framework of the COST Action MP0805, headed by our dear friend, the late Prof. Naci Balkan. We acknowledge funding from Programme Investissements d'Avenir ANR-11-IDEX-0002-02, reference ANR-10- LABX-0037-NEXT. X.M. also acknowledges Institut Universitaire de France. The growth part has been carried out in the LAAS platform, a member of the French Renatech network. 


\section{Figure Caption :}

\section{Figure 1}

Low temperature ( $T=10 \mathrm{~K}$ ) normalized photoluminescence spectra for a) three $\mathrm{GaAs}_{1-\mathrm{x}} \mathrm{Bi}_{\mathrm{x}}$ quantum well structures $\left(\mathrm{L}_{\mathrm{W}}=7 \mathrm{~nm}\right)$ with $\mathrm{Bi}$ content $\mathrm{x}=2.4 \%, 5.5 \%$ and $7 \%$ and b) several GaAsBi epilayer with Bi content ranging from $1.16 \%$ to $3.83 \%$.

\section{Figure 2}

Photoluminescence intensity dynamics of the a) $2.4 \%$ and b) $7 \% \mathrm{GaAs}_{1-\mathrm{x}} \mathrm{Bi}_{\mathrm{x}} / \mathrm{GaAs}$ quantum well structures as a function of the temperature.

\section{Figure 3}

a) Photoluminescence circular polarization dynamics in three quantum well structures with different $\mathrm{Bi}$ contents $(\mathrm{x}=2.4 \%, 5.5 \%, 7 \%) . \mathrm{T}=150 \mathrm{~K}$. The lines are guides to the eye. $\mathrm{b}$ ) Corresponding temperature dependence of the spin relaxation time $\tau_{s}$.

\section{Figure 4}

a) Photoluminescence circular polarization dynamics in bulk $\mathrm{GaAs}_{1-\mathrm{x}} \mathrm{Bi}_{\mathrm{x}}$ for $\mathrm{x}=1.16 \%, 1.8 \%$, 2.34\%, 3.04\% and 3.83\%. $\mathrm{T}=100 \mathrm{~K}$. b) Corresponding temperature dependence of the spin relaxation time $\tau_{s}$. 
Table 1

\begin{tabular}{c|c|c|c|}
\hline \multicolumn{2}{c|}{ Bulk layers } & \multicolumn{2}{c|}{ Quantum wells } \\
\hline Thickness $(\mathrm{nm})$ & $\mathrm{Bi}(\%)$ & Thickness $(\mathrm{nm})$ & $\mathrm{Bi}(\%)$ \\
\hline 233 & 1,16 & 7,7 & 2,42 \\
\hline 230 & 1,8 & 7,5 & 5,5 \\
\hline 258 & 2,34 & 7,6 & 6,98 \\
\hline 147 & 3,04 & - & - \\
\hline 265 & 3,83 & - & - \\
\hline
\end{tabular}


Figure 1
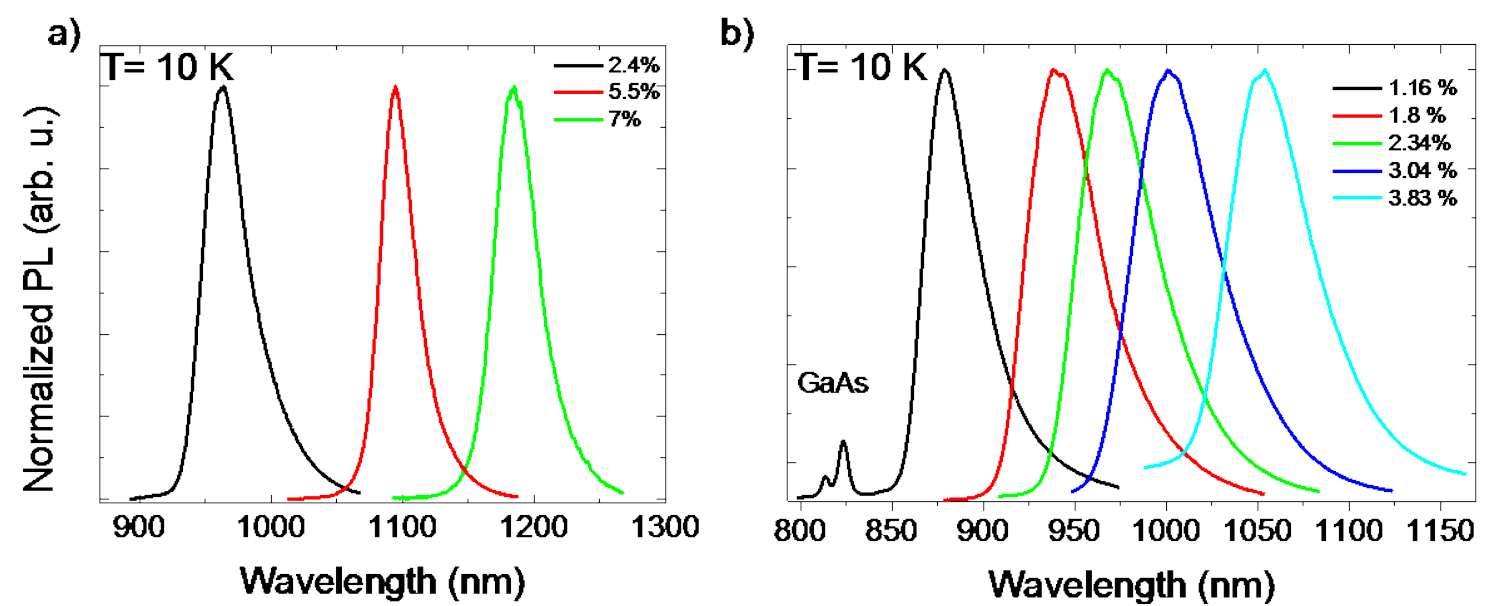

Figure 2
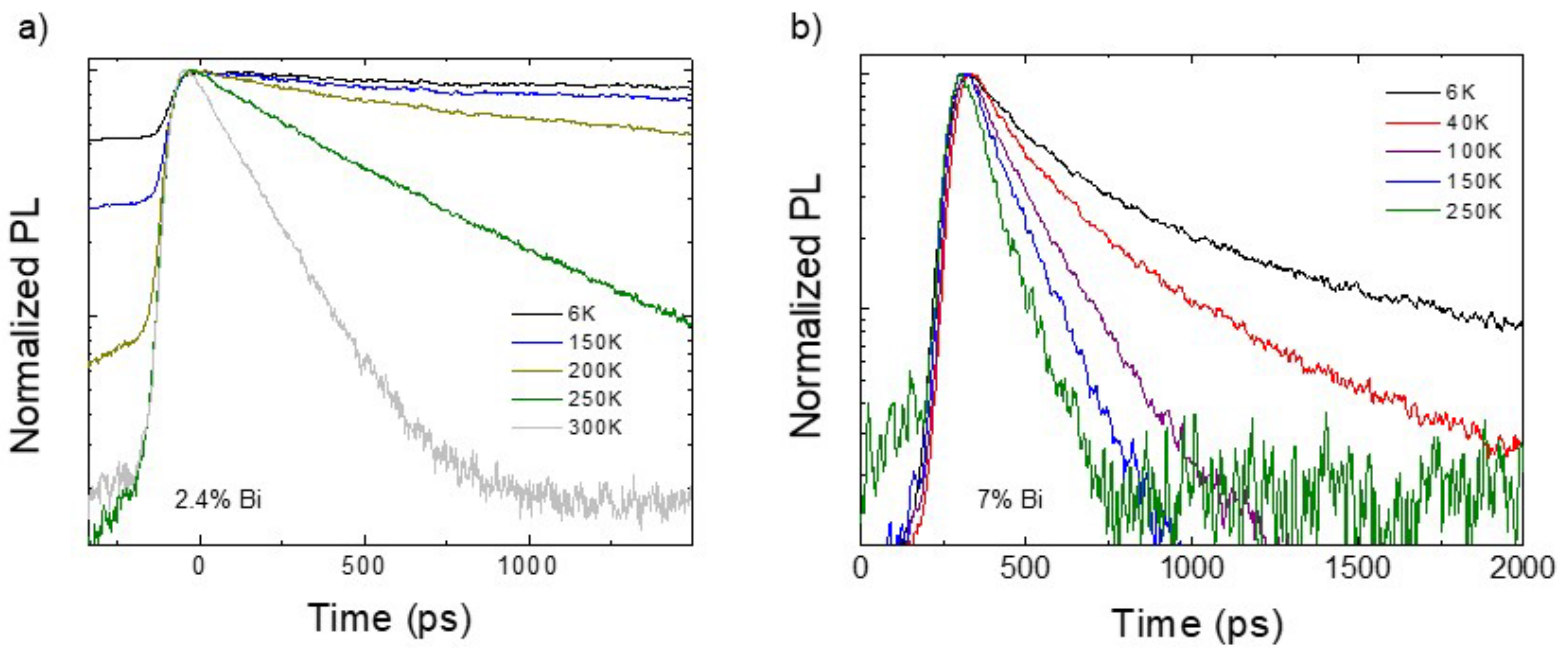


\section{Figure 3}

a)

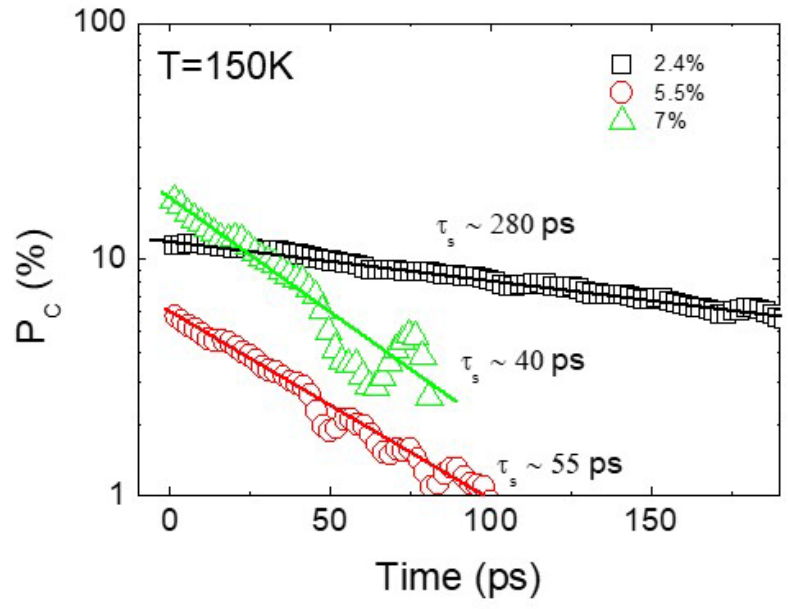

b)

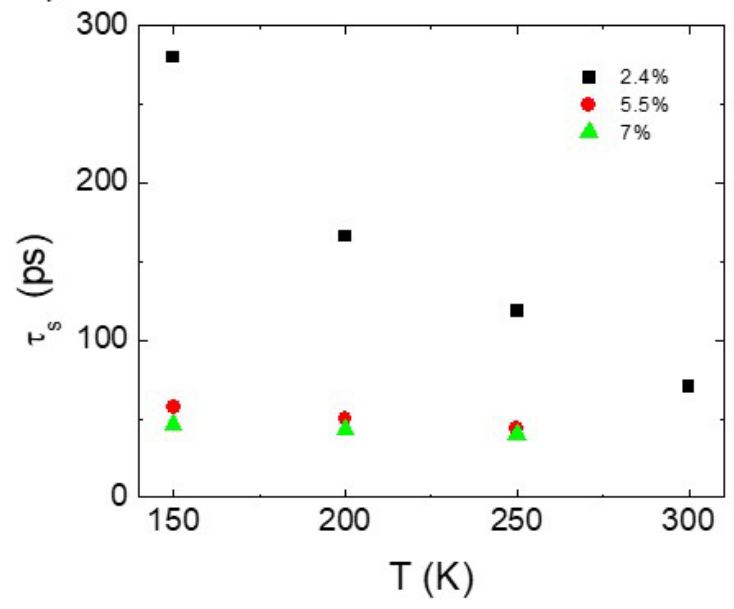

\section{Figure 4}
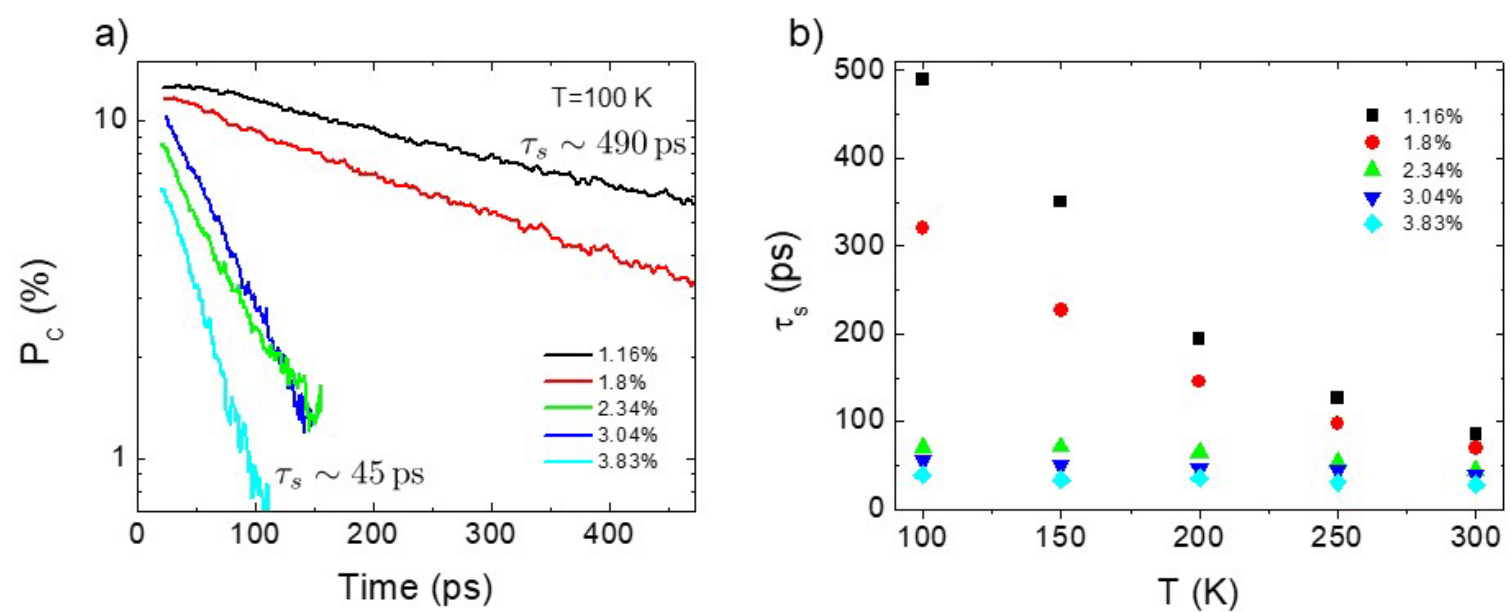

\section{References :}

\footnotetext{
${ }^{1}$ S. Tixier, A. Adamcyk, A. Tiedje, S. Francoeur, A. Mascarenhas, P. Wei and F. Schiettekatte, Appl. Phys. Lett. 82, 2245 (2003).
}

${ }^{2}$ M.Yoshimoto, S.Murata, A.Chayahara, Y. Horino, J. Saraie, K. Oe , Jpn. J. Appl. Phys. 42, 10B (2003)

${ }^{3}$ K. Bertulis, A. Krotkus, G. Aleksejenko, V. Pac`ebutas, R. Adomavic`ius, G. Molis, and S. Marcinkevic`ius, Appl. Phys. Lett. 88, 201112 (2006).

${ }^{4}$ R.D. Richards, A. Mellor, F. Harun, J.S. Cheong, N.P. Hylton, T. Wilson, T. Thomas, J.S. Roberts, N.J. EkinsDaukes, J.P.R. David, Solar Energy Materials and Solar Cells 172, 238 (2017)

${ }^{5}$ B. Fluegel, S. Francoeur, A. Mascarenhas, S. Tixier, E. C. Young, and T. Tiedje, Phys. Rev. Lett. 97, 067205 (2006). 
${ }^{6}$ K. Alberi, O. D. Dubon, W. Walukiewicz, K. M. Yu, K. Bertulis, and A. Kroktus, Appl. Phys. Lett. 91, 051909 (2007).

${ }^{7}$ S. R. Jin, S. J. Sweeney, J. Appl. Phys., 114, 21310 (2013)

${ }^{8}$ I. P. Marko, J. S. Sweeney, IEEE J. Select. Topics Quantum Elect. 23, 1501512 (2017)

${ }^{9}$ S. Mazzucato, T. T. Zhang, H. Carrère, D. Lagarde, P. Boonpeng, A. Arnoult, G. Lacoste, A. Balocchi, T. Amand, C. Fontaine, and X. Marie, Applied Phys. Lett. 102, 252107 (2013)

${ }^{10}$ C. A. Broderick, S. Mazzucato, H. Carrère, T. Amand, H. Makhloufi, A. Arnoult, C. Fontaine, O. Donmez, A. Erol, M. Usman, E.P. O’Reilly, and X. Marie, Phys. Rev. B 90, 195301 (2014)

${ }^{11}$ M. A. G. Balanta, J. Kopaczek, V. Orsi Gordo, B. H. B. Santos, A. D. Rodrigues, H. V. A. Galeti, R. D. Richards, F. Bastiman, J. P. R. David, R. Kudrawiec, Y. Galvão Gobato, J. Phys. D: Appl. Phys., 49, 355104 (2016)

${ }^{12}$ H. Tong, X. Marie, and M. W. Wu, J. Appl. Phys. 112, 063701 (2012)

${ }^{13}$ B. Pursley, M. Luengo-Kovac, G. Vardar, R. S. Goldman, and V. Sih, Appl. Phys. Lett. 102, 022420 (2013)

${ }^{14}$ M. I. D’yakonov and V. I. Perel', Fiz. Tverd. Tela 13, 3581 (1971)[Sov. Phys. Solid State 13, 3023 (1972)] ; M. I. D’yakonov and V. Yu. Kachorovskii, Fiz. Tekh. Poluprovodn. 20, 178 (1986)[Sov. Phys. Semiconductors 20, 110 (1986)].

${ }^{15}$ G. Dresselhaus, Phys. Rev. 100, 580 (1955)

${ }^{16}$ A. Tackeuchi, O. Wada, Y. Nishikawa Appl. Phys. Lett. 70, 1133 (1997)

${ }^{17}$ M. W. Wu, J. H. Jiang, and M. Q. Weng, Phys. Rep. 493, 61 (2010).

${ }^{18}$ A. Malinowski, R. S. Britton, T. Grevatt, R. T. Harley, D. A. Ritchie and M. Y. Simmons, Phys. Rev. B 62, 13034 (2000)

${ }^{19}$ A. Balocchi, Q. H. Duong, P. Renucci, B. L. Liu, C. Fontaine, T. Amand, D. Lagarde, and X. Marie, Phys. Rev. Lett. 107, 136604 (2011)

${ }^{20}$ G. L. Bir, A. G. Aronov, and G. E. Pikus. Spin relaxation of electrons due to scattering by holes. Sov. Phys. JETP, 42:705, 1975

${ }^{21}$ R. J. Elliott. Theory of the e ffodtiof spiping on magnetic resonance in some semiconductors. Phys. Rev., 100:266, 1954

${ }^{22}$ K. L. Litvinenko, M. A. Leontiadou, J. Li, S. K. Clowes, M. T. Emeny, T. Ashley, C. R. Pidgeon, L. F. Cohen, and B. N. Murdin, Appl. Phys. Lett. 96, 111107 (2010)

${ }^{23}$ J. H. Jiang and M. W. Wu, Phys. Rev. B 79, 125206 (2009)

${ }^{24}$ J. H. Jiang and M. W. Wu, Phys. Rev. B 83, 239906 (2011)

${ }^{25}$ H. Makhloufi, P. Boonpeng, S. Mazzucato, J. Nicolai, A. Arnoult, T. Hungria, G. Lacoste, C. Gatel, A. Ponchet, H., X. Marie and C. Fontaine, Nanoscale Research Lett. 9,123 (2014)

${ }^{26}$ L. J. Sham, I. Phys.: Condens. Matter 5 A51 (1993) 
${ }^{27}$ B. Baylac, T. Amand, X. Marie, B. Dareys, M. Brousseau, G. Bacquet, V. Thierry-Mieg, Sol. Stat. Comm. 93, 57 (1995)

${ }^{28}$ D. J. Hilton, C.L.Tang, Phys.Rev.Lett. 89,146601 (2002)

${ }^{29}$ F. Meier and B.P. Zakharchenya, Optical Orientation (Elsevier Science Ltd., New York, 1984)

${ }^{30}$ Z. Batool, K. Hild, T. J. C. Hosea, X. Lu, T. Tiedje, and S. J. Sweeney, J. Appl. Phys. 111, 113108 (2012)

${ }^{31}$ S. J. Sweeney and S. R. Jin, J. Appl. Phys. 113, 043110 (2013)

${ }^{32}$ S. Mazzucato, H. Lehec, H. Carrère, H. Makhloufi, A. Arnoult, C. Fontaine, T. Amand, X. Marie, Nanoscale Res. Lett. 9, 19 (2014)

${ }^{33}$ S. Mazzucato, P. Boonpeng, H. Carrère, D. Lagarde, A. Arnoult, G. Lacoste, T. Zhang, A. Balocchi, T. Amand, X. Marie, Semicond. Sci. Technol. 28, 022001 (2013)

${ }^{34}$ D. G. Cooke, F. A. Hegmann, E. C. Young, T. Tiedje, Appl. Phys. Lett. 89, 122103 (2006)

${ }^{35}$ R. N. Kini, L. Bhusal, A. J. Ptak, R. France, A. Mascarenhas, J. Appl. Phys. 106, 043705 (2009); R. N. Kini and A. Mascarenhas, (2013) Effect of Bismuth Alloying on the Transport Properties of the Dilute Bismide Alloy, GaAs ${ }_{1-x} \mathrm{Bi}_{x}$. In: Li H., Wang Z. (eds) Bismuth-Containing Compounds. Springer Series in Materials Science, vol 186. Springer, New York, NY

${ }^{36}$ V. Pacebutas, K. Bertulis , L. Dapkus, G. Aleksejenko, A. Krotkus, K. M. Yu, W. Walukiewicz, Semicond. Sci. Technol. 22, 819 (2007)

${ }^{37}$ A. Urbanowicz, V. Pačebutas, A. Geižutis, S. Stanionytė, A. Krotkus, AIP Advances 6, 025218 (2016)

${ }^{38}$ B. Fluegel, R. N. Kini, A. J. Ptak, D. Beaton, K. Alberi, A. Mascarenhas, Appl. Phys. Lett. 99, 162108 (2011)

${ }^{39}$ G. Pettinari, A. Polimeni, M. Capizzi, J. H. Blokland, P. C. M. Christianen, J. C. Maan, E. C. Young, and T. Tiedje, Appl. Phys. Lett. 92, 262105 (2008)

${ }^{40}$ G. Pettinari, O. Drachenko, R. B. Lewis, T. Tiedje, Phys. Rev. B 94, 235204 (2016)

${ }^{41}$ M. Usman, C. A. Broderick, A. Lindsay, E. P. O’Reilly, Phys. Rev. B 84, 245202 (2011)

${ }^{42}$ R. Kudrawiec, J. Kopaczek, M. P. Polak, P. Scharoch, M. Gladysiewicz, J. Misiewicz, R. D. Richards, F. Bastiman, J. P. R. David, J. Appl. Phys. 116, 233508 (2014)

${ }^{43}$ J. Kopaczek, W. M. Linhart, M. Baranowski, R. D. Richards, F. Bastiman, J. P. R. David, R. Kudrawiec, Semicond. Sci. Technol. 30, 094005 (2015)

${ }^{44}$ P. Ludewig, N. Knaub, N. Hossain, S. Reinhard, L. Natterman, I. P. Marko, S. R. Jin, K. Hild, S. Chatterjee, W. Stolz, S. J. Sweeney, K. Volz, Appl. Phys. Lett. 102, 242115 (2013)

45 S. Nagahara, M. Arita, Y. Arakawa, Appl. Phys. Lett. 88, 083101 (2006)

${ }^{46}$ J. Puustinen, M. Wu, E. Luna, A. Schramm, P. Laukkanen, M. Laitinen, T. Sajavaara, M. Guina, J. Appl. Phys. 111, 243504 (2013); M. Wu, E. Luna, J. Puustinen, M. Guina, A. Trampert, Nanotechnology 25, 205605 (2014)

${ }^{47}$ D. A. Beaton, M. Steger, A. Mascarenhas, J. Cryst. Growth 484, 7 (2018)

${ }^{48}$ C. R. Tait, L. Yan, J. M. Millunchick, J. Cryst. Growth 493, 20 (2018) 
${ }^{49}$ F. T. Vasko F T, JETP Lett. 30, 541 (1979) ; Y. A. Bychkov, E. Rashba JETP. Lett. 39, (1984) ; Y. A. Bychkov, E. Rashba, J. Phys. C: Solid State Phys. 17, 6039 (1984)

${ }^{50}$ Y. Ohno, R. Terauchi, T. Adachi, F. Matsukura, H. Ohno, Phys. Rev. Lett. 83, 4196 (1999)

${ }^{51}$ O. Z. Karimov, G. H. John, and R. T. Harley, W. H. Lau, M. E. Flatte, M. Henini, R. Airey, Phys. Rev. Lett. 91, 246601 (2003)

52 S. Döhrmann, D. Hägele, J. Rudolph, M. Bichler, D. Schuh, and M. Oestreich

Phys. Rev. Lett. 93, 147405 (2004)

53 G. Wang, A. Balocchi, A. V. Poshakinskiy, C. R. Zhu, S. A. Tarasenko, T. Amand, B. L. Liu and X. Marie, New Journal of Physics 16, 045008(2014)

${ }^{54}$ A. Balocchi, T. Amand, G. Wang, B. L. Liu, P. Renucci, Q. H. Duong, X. Marie, New J. Phys. 15,095016 (2013)

${ }^{55}$ G. Wang, B.L. Liu, A. Balocchi, P. Renucci, C.R. Zhu, T. Amand, C. Fontaine, X. Marie, Nat. Comm., DOI: 10.1038/ncomms3372 (2013)

${ }^{56}$ S. Azaizia, A. Balocchi, H. Carrère, P. Renucci, T. Amand, A. Arnoult, C. Fontaine, X. Marie, Appl. Phys. Lett. 108, 082103 (2016)

${ }^{57}$ R. A. Simmons, S. R. Jin, S. J. Sweeney, S. K. Clowes, Appl. Phys. Lett. 107, 142401 (2015) 